\title{
Enseigner le genre à des apprenants allophones
}

Réflexions et propositions didactiques pour un enseignement non sexiste du FLE

- Insegnare il genere a discenti allofoni. Riflessioni e proposte didattiche per un insegnamento non sessista in FLE

\section{MANCONI Thérèse et SHEEREN Hugues}

\section{(2) OpenEdition \\ Journals}

Édition électronique

URL : https://journals.openedition.org/rdlc/8278

DOI : $10.4000 /$ rdlc. 8278

ISSN : $1958-5772$

Éditeur

ACEDLE

\section{Référence électronique}

MANCONI Thérèse et SHEEREN Hugues, "Enseigner le genre à des apprenants allophones »,

Recherches en didactique des langues et des cultures [En ligne], 17-3 | 2020, mis en ligne le, consulté le 20 juillet 2021. URL : http://journals.openedition.org/rdlc/8278 ; DOI : https://doi.org/10.4000/rdlc. 8278

Ce document a été généré automatiquement le 20 juillet 2021.

\section{(c) $(1) \odot$}

Recherches en didactique des langues et des cultures is licensed under a Creative Commons AttributionNonCommercial-NoDerivatives 4.0 International License 


\title{
Enseigner le genre à des apprenants allophones
}

\author{
Réflexions et propositions didactiques pour un enseignement non sexiste \\ du FLE ${ }^{1}$ \\ - Insegnare il genere a discenti allofoni. Riflessioni e proposte didattiche \\ per un insegnamento non sessista in FLE
}

\section{MANCONI Thérèse et SHEEREN Hugues}

\section{Introduction}

« En grammaire comme ailleurs, les analyses ne sont jamais

achevées ni les réponses définitives. » (Riegel et al. : 1994)

1 Les avancées documentées et avérées dans l'acquisition des langues étrangères semblent avoir peu ou prou pénétré les dispositifs de description de la langue («la grammaire ») des manuels, des méthodes, des approches (même actionnelles ou communicatives) tant en FLE qu'en FLM. La grammaire dite normative ${ }^{2}$, dont les limites ont été depuis largement attestées, continue de représenter l'état de la langue. Ainsi en va-t-il de la description genrée de la morphologie / flexion nominale et adjectivale et également de la féminisation des titres, fonctions et professions.

2 Les polémiques suscitées à la parution, fin septembre 2017, d'un manuel adoptant l'écriture inclusive chez $\mathrm{Hatier}^{3}$, ainsi que la virulence du débat politique et sociétal que cela a pu déclencher nous confirment la portée symbolique que toute "atteinte » (supposée, viscérale et somme toute peu rationnelle) à l'écriture du français semble ébranler ou « menacer $»^{4}$.

3 Soyons clairs : ces représentations ne sont pas les nôtres; nous allons tenter de le démontrer par nos pratiques d'enseignement.

4 Nous nous rangeons, de manière claire aux côtés d'Éliane Viennot et des termes de sa pétition ${ }^{5}$ ainsi que de son ouvrage; mais aussi du guide très utile publié par Anne Dister et Marie-Louise Moreau (2020). Il nous parait également important de citer le numéro 
11 de la revue Langue et discours (Rabatel et Rosier, 2019), entièrement consacré au débat sur l'écriture inclusive, et dont la lecture nous parait incontournable pour entamer une réflexion sur « les défis de l'écriture inclusive ».

5 Par ailleurs, les débats actuels concernant l'écriture inclusive voire l'accord de proximité semblent bien pour le moment être délaissés par la didactique du français ${ }^{6}$. Dans une société où les pratiques langagières, qu'on le veuille ou non, changent, n'y a-til pas lieu - de sensibiliser les apprenantes et apprenants ${ }^{7}$ au phénomène sociétal qui voit les femmes conquérir et revendiquer - à juste titre - davantage d'espace dans la communication quotidienne, davantage de visibilité, y compris à travers les pratiques langagières? Mieux, pourquoi ne pas enseigner ces nouvelles formes?

Notre contribution se fixe pour objectif de poser de nouvelles balises pour l'acquisition en FLE, de la flexion nominale/adjectivale féminin vs masculin, ainsi que d'amener les apprenant.e.s vers un processus réflexif (métalangagier) quant aux questions du genre, à travers la féminisation des titres, fonctions et professions, mais également sur la pertinence de l'écriture inclusive, sous toutes ses formes (choix d'un terme épicène, doublet intégral, point médian, barre oblique...). Nous illustrerons donc, dans un premier temps, une démarche pédagogique adoptée dans le cadre de cours universitaires de FLE pour l'acquisition de la formation du masculin à partir du féminin (et non l'inverse). Cela nous autorisera quelques réflexions sur la pertinence et l'efficacité de nouveaux modèles de description de la langue au profit des compétences communicatives.

7 Dans un second temps, nous montrerons de quelle manière nous cherchons à diffuser les termes féminins relatifs à des professions ou titres récemment entrés dans la langue et nous verrons aussi comment et pourquoi nous cherchons à rompre le cloisonnement masculin/féminin. Ceci nous amènera ensuite à rendre compte des stratégies langagières que nous mettons en œuvre concrètement dans notre travail quotidien pour donner plus de visibilité aux femmes, tant dans notre matériel écrit que dans nos interactions orales avec la classe.

\section{Le féminin et le masculin : le morphologique/ phonologique au service des compétences de communication}

8 Nous le savons déjà : la "tradition grammaticale " explique que le féminin se forme à partir du masculin. Le féminin y est présenté comme une forme "fléchie ", "dérivée ", " altérée » (Lenoble-Pinson, 2006: 643). En témoignent les titres des chapitres de nombreux manuels de FLE comme "le féminin », «la formation du féminin », laissant supposer que le masculin est donné pour acquis puisqu'il se situe du côté de la régularité, de la norme, de la stabilité et que le féminin en découle.

Cette «logique» est transmise depuis des générations et n'affecte pas uniquement la langue française. Elle est sans doute à mettre en lien direct avec la version la plus connue du récit de la Genèse relatant qu'Ève est née de la côte d'Adam. Elle est le reflet d'une vision "scriptocentrée » de la langue, qui tend à expliquer de manière simpliste la règle de formation du féminin (ajout d'un $e$ à l'écrit) alors qu'à l'oral, nous le savons, le procédé est bien plus complexe, comme nous le verrons plus loin. À l'instar de la règle « le masculin l'emporte sur le féminin » qui continue à être transmise oralement 
au point d'être presque devenue une expression proverbiale alors qu'elle a disparu des manuels depuis la fin du $19^{\mathrm{e}}$ siècle, cette démarche est sans aucun doute révélatrice d'une vision patriarcale de la langue.

10 Les outils dont disposent les enseignant $\backslash e \backslash s$ de FLE adoptent, sans remettre en question, ce procédé «logique». Ainsi en va-t-il, par exemple, de la Grammathèque ${ }^{8}$, grammaire contrastive très répandue en Italie, qui propose plusieurs pages où les adjectifs et les substantifs au féminin sont illustrés en partant des formes masculines, même si certains nouveaux féminins sont mis en évidence, accompagnés de quelques remarques. Corollaire de cette manière d'expliquer la règle: dans les exercices proposés, on demande, la plupart du temps, aux étudiant-e-s de "former le féminin ", "d'accorder ", « de transformer » ou de "mettre » au féminin, à l'exception d'un seul exercice où il est demandé de «mettre au masculin ». De même, La Nouvelle Grammaire $d u$ Français. Cours de Civilisation Française de la SORBONNE (2004), n'est malheureusement pas aussi innovante que le titre ne pourrait le laisser supposer puisqu'elle enseigne la formation du féminin et énonce la règle «on ajoute un -e à la forme écrite du masculin ».

11 Du collège au lycée, la démarche est invariablement la même. Dans le manuel Vitamines (2010), destiné aux 11-14 ans, les exercices se présentent invariablement sous la forme canonique. Il s'agit de mettre au féminin des phrases comme Il est grand et gros ou Il est petit et brun (Crimi-Hatuel, $2010: 32$ ) voire un petit texte : "Je m'appelle Adrien et j'ai un chien, il s'appelle Caramel. Il est beau, il est gentil [...] ». Il est demandé de réécrire le texte avec une chatte qui s'appelle Vanille, qui est belle, gentille, etc. (Crimi-Hatuel, 2010 : 48). Une approche similaire est proposée dans la Grammaire progressive du français (2017), qui, bien qu'elle nomme un sous-titre de chapitre de façon neutre (« Le nom : masculin et féminin des personnes/des choses »), propose ensuite essentiellement des exercices où il est demandé de transformer de courtes phrases du masculin au féminin.

Remarquons, toutefois, l'approche sensiblement différente de la Grammaire du français pour italophones de Françoise Bidaud (2012) : on envisage, à juste titre, «le genre » (et non pas le féminin) et on peut y lire des remarques pertinentes sur la féminisation des noms de professions et fonctions. Néanmoins, l'approche globale n'échappe pas à la tradition.

13 Pour sortir quelque peu de cette vision ritualisée et sacralisée de la langue, Thérèse Manconi propose dans le cadre de ses cours une approche «inversée », fondée sur la forme-étalon prônée par Bargy (1925) pour la description phonétique de l'indicatif présent ${ }^{9}$.

14 En effet, Bargy, propose avant tout de ne pas ramener le simple au complexe. Dans sa "grammaire de l'oreille », la «forme-étalon » du verbe est la forme complète. Nous la reprenons à notre compte et pour le propos qui est le nôtre. Relisons Bargy :

«[...] On morcèle la langue en une féodalité de verbes dits irréguliers. En fait, s'il y a de l'irrégularité, elle est dans l'Infinitif. C'est lui la forme-exception. [...] Y rapporter les autres, c'est ramener le simple au complexe. [...] Tandis qu'il n'importe guère de formuler des lois de l'Infinitif et du Participe passé, il y a urgence à asseoir une théorie du Présent sur une définition précise de ses formes. Dans une "grammaire de l'oreille", toute définition de forme est une définition phonétique. Exemple : "je dors" n'est pas défini par l's muet mais par l'absence de l'm de "dorment". [...] Par comparaison avec la $3^{\mathrm{e}}$ personne pluriel, les formes "sens, sers, émeus, sais, vaux, crains, viens, etc.", aussi bien que les formes "finis, vis, reçois, etc. ", se définissent par la chute 
du son-consonne final, qui est le fait fondamental et général. [...] Le radical du verbe est le son de la $3^{\mathrm{e}}$ personne pluriel. [...] La $3^{\mathrm{e}}$ personne pluriel est l'incarnation vivante du radical. Elle fait du radical une réalité : par elle, il chante dans l'oreille et reste dans la mémoire; par elle, la relation entre le son du radical et le son de n'importe quelle forme est un fait musical clair » (Bargy, ib. : 13-18-32).

Nous pointerons ici la priorité accordée à la relation « entre le son [du radical] et le son de n'importe quelle forme (...)». Notre démarche annoncée considère donc que la forme-étalon est celle du féminin. Dans la pratique d'enseignement du Français Langue Étrangère à des étudiant.e.s de Lingue e Culture moderne (à l'Université de Pavie ou de Scienze della mediazione interlinguistica e interculturale (Università dell'Insubria), nous avons proposé un modèle de description de la morphologie du féminin (mais pas seulement) se basant sur la forme-étalon, à savoir la forme que prend (à l'oral d'abord, à l'écrit ensuite) le féminin du nom ou de l'adjectif. La formation du masculin s'explique donc par un passage de ce type :

Elle est française. > Il est français.

C'est une étudiante. > C'est un étudiant.

Contrairement à l'option majoritaire choisie par les grammaires, les manuels, les méthodes de FLE (voire de FLM), le modèle proposé ici vise à mettre en avant le son (la forme orale et la pertinence des informations qu'elle véhicule en termes de genre), sans pour autant occulter ou évacuer l'écrit (nous reviendrons plus loin sur l'efficacité de ce modèle en termes de "rentabilité orthographique»). Le rapport entre écriture et prononciation s'enrichit, selon nous, d'un point de vue nouveau : envisager le masculin à partir du féminin, par le biais du mécanisme de l'élision que nous empruntons à Bargy, comme nous l'avons indiqué supra.

Le recours au point médian valorise, selon nous, encore davantage la pertinence double de ce " graphème " : à l'oral et à l'écrit. Ceci sans préjuger des difficultés de lecture que certain.e.s opposant.e.s à l'écriture inclusive avancent mais, toutefois, dans l'attente de preuves rigoureuses des "complications" réelles que tel point médian engendrerait dans les stratégies de mise en place d'une compétence de lecture. Affaire à suivre, donc.

Qu'il nous soit donc permis de fixer le point central de notre démarche : le masculin se définit par l'élision, à l'oral, de la consonne finale présente au féminin. Ce faisant, l'attention de l'apprenant.e se focalise prioritairement sur la pertinence de l'information portée par la consonne finale prononcée, mais également sur la pertinence, à l'écrit, de la présence (ou élision/absence) de la désinence -e. Oral et écrit peuvent ainsi se compléter.

\section{Considérations annexes et corollaires}

19 Une fois entérinée et mise à l'épreuve, notre démarche autorise quelques considérations annexes, que nous déclinerons, dans le champ phonologique, autour de trois points considérés comme « sensibles » dans la pratique du FLE :

- Nasalisation et dénasalisation

- Rapport entre oral(ité) et écrit(ure)

- Réalisation / non réalisation de la consonne finale 
En effet, de simples observations de productions (orales ou écrites) de nos apprenante.s allophones (pour nous, majoritairement italophones) mettent en évidence les difficultés que ceux-/celles-ci rencontrent dans l'acquisition à la fois du système phonologique $d u$ français mais également du rapport non univoque entre écriture et prononciation. Nous nous attarderons en particulier sur les points annoncés supra.

\section{Nasalisation / dénasalisation : italien prononcé comme italienne}

21 Le passage de italienne à italien est présenté en mettant en évidence le mécanisme selon lequel le /n/ est, certes, lui aussi élidé mais il en reste une trace par le biais de la nasalisation de la voyelle qui précède. Ceci représente également un avantage certain (que nous avons pu vérifier) en termes de technique articulatoire. Ainsi, bonne/bon, américaine/américain. Pour fine/fin, une explication ultérieure sera nécessaire, mais tel n'est pas notre propos ici.

\section{De l'oral (ité) à l'écrit (ure)}

On l'aura compris : dans les premières phases d'apprentissage/d'enseignement, l'oral (l'oralité) a la priorité dans les activités proposées aux apprenant.e.s; le passage à l'écrit (l'écriture) consiste à vérifier que la transcription des morphèmes en graphèmes est pertinente (quitte à accepter, dans des phases transitoires, des approximations en rien dommageables : ainsi *italiene au lieu de italienne). Nous évacuons, pour l'instant, (cf. supra) toute considération sur les difficultés supposées à « lire » l'écriture inclusive (difficultés que, nous le répétons ici, nous n'avons pas rencontrées chez nos apprenant.e.s).

\section{Réalisation / non-réalisation d'une consonne finale : avocat prononcé comme avocate}

Que le graphème - $t$ soit déjà présent au masculin ne facilite guère les choses pour nos apprenant.e.s (majoritairement italophones et donc détenteurs d'une tradition orthographique dans leur langue maternelle hautement plus prédictible que pour leurs homologues francophones). Le fait, en plus, que la grammaire traditionnelle se limite à dire que le féminin des noms et des adjectifs se forme par l'adjonction d'un -e, entérine définitivement l'incohérence du système orthographique du français et explique, du moins en partie, les «ratés " dans la maitrise de l'orthographe pré- ou post-réforme (aussi bien chez des scripteurs/trices natifs/ves que non natifs/ves : la convergence des résultats autoriserait, sur ce point, une réflexion commune en didactique du FLE et du FLM). Comment, en effet, ne pas voir une contradiction orthographique entre des termes comme lettone et baronne, ou entre artisane et paysanne? En enseignant le féminin selon l'optique traditionnelle, on est contraint de compléter la règle par une liste $d$ ' « exceptions » si longue que la question du bien-fondé de la règle même se pose tout naturellement.

Nous relèverons surtout que la formation du masculin, telle que nous l'avons énoncée, met en valeur le(s) phénomène(s) en jeu, tant à l'oral qu'à l'écrit. Nous appliquons cette approche ailleurs aussi car elle permet, en effet, de rendre compte autrement, selon un point de vue fondé d'après Bargy, des doubles masculins comme nouveau/nouvel, beau/ 
bel, fou/fol, mou/mol et vieux/vieil. En partant du féminin, les élèves appréhendent ces formes dans un schéma visant davantage à illustrer la régularité, plutôt que l'irrégularité et, par conséquent, les mémorisent mieux. Une procédure que nous préconisons également pour le déterminant démonstratif masculin $c e / c e t$. La double forme masculine est, par ailleurs, souvent source d'erreurs.

\section{Ramener le complexe au simple : adapter n'est pas adopter}

Toutes les activités proposées sont le fruit d'un travail personnel. Il nous est arrivé, toutefois, de détourner quelques exercices n'étant pas de notre cru et de les "plier » à nos exigences. En particulier, ceux de la collection Phonétique progressive du français (2015), publié chez CLE International.

Exemple d'exercice : nous demandons aux apprenantes et apprenants de compléter un tableau comme celui présenté ci-dessous, en relevant, à la fois les phénomènes apparaissant à l'oral et ceux apparaissant à l'écrit.

\begin{tabular}{|c|c|}
\hline Féminin $\neq$ Masculin $^{10}$ & \\
\hline Elle est étudiante. /t/ & Il est étudiant. / / \\
\hline C'est la boulangère. / $\varepsilon r /$ & C'est le boulanger. /e/ \\
\hline Elle est française. /z/ & Il est français. / / \\
\hline Je suis italienne. /عn/ & Il est italien. $/ \varepsilon 7$ \\
\hline Elle est très gentille. /ij/ & Il est très gentil. /i/ \\
\hline Elle est avocate. & Il est __-_-_-_-_. \\
\hline Elle est pharmacienne. & Il est \\
\hline Elle est__ & Il est infirmier. \\
\hline
\end{tabular}

Nota bene: L'apparition/disparition de l'accent graphique (comparer: C'est la boulangère. $/ \varepsilon \mathrm{r} / \mathrm{vs} \mathrm{C}^{\prime}$ est le boulanger. /e/) est traité selon les règles du vocalisme en français (en syllabe tonique ouverte, voyelle fermée ; en syllabe tonique fermée, voyelle ouverte), non sans avoir proposé des activités sur le découpage syllabique graphique vs découpage syllabique oral.

\section{Quelques commentaires}

Comme nous l'avons précisé plus haut, il ne s'agit nullement d'évacuer l'écrit, mais bien au contraire de ne l'envisager qu'une fois que les formes orales sont assurées. Dans ce sens, il nous semble que les critères d'évaluation des productions orales et des productions écrites dans le cadre des certifications du DELF (niveau B1) nous confortent dans notre option. Nous pointons ci-dessous quelques fragments des grilles d'évaluation de ces épreuves (élaborées par le CIEP et disponibles en ligne à l'adresse 
suivante : http://www.ciep.fr/sites/default/files/migration/delfdalf/documents/ DELF_B1.pdf)

Pour la production écrite

\begin{tabular}{|l|l|l|l|l|l|}
\hline $\begin{array}{l}\text { Morphosyntaxe - orthographe grammaticale } \\
\text { Accord en genre et en nombre, pronoms, marques verbales, etc. }\end{array}$ & 0 & 0,5 & 1 & 1,5 & 2 \\
\hline
\end{tabular}

Figure 1

Pour la production orale

Figure 2

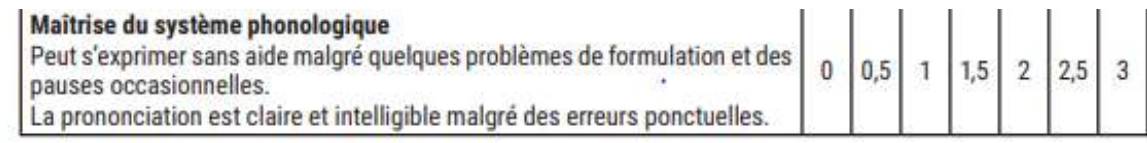

L'espace nous manque pour fournir, dans le cadre de cet article, un corpus important de résultats permettant de comparer les performances des étudiant.e.s lors du test de niveau initial, en début d'année académique et lors des productions orales et écrites de fin de module, au terme du premier semestre. Nous pouvons néanmoins attester de la stabilisation et de l'autonomisation consolidées à travers cette approche en termes d'efficacité et de rentabilité, même " orthographique ».

\section{Enseigner à féminiser davantage}

Depuis environ trente ans, les pays francophones où sont présentes des institutions qui prennent des décisions en matière de langue (Belgique, France, Québec et Suisse) ont émis des propositions concernant la féminisation des titres, grades, fonctions et métiers. Qu'elle qu'en soit la forme ou l'appellation (décret, recommandations, circulaire, guide...), ces instances ont pris clairement position, même si cela se fit à des degrés divers, en faveur d'une plus grande visibilité de la femme dans la langue par le biais de la féminisation de substantifs ayant trait à la place qu'occupent les femmes dans le monde professionnel.

« La féminisation des noms de métiers, titres et fonctions illustre à merveille cette autonomie francophone, et le laborieux deuil français; car c'est en périphérie que tout a commencé. C'est-à-dire au sein de ce qu'une morgue hexagonale tient pour une appropriation marginale, incorrecte, voire illicite de la langue »(Cerquiglini, $2018: 15)$.

Alors que les maisons d'édition n'ont de cesse de se "mettre à jour ", acte souvent purement commercial, les manuels de FLE sont encore à la traine. Trop frileux, ils n'enseignent malheureusement que rarement des formes féminines aujourd'hui pourtant bien présentes dans la langue. Qu'elles soient de facture italienne ou française, les méthodes de français destinées aux personnes étrangères diffusent encore trop peu de termes qui sont néanmoins rentrés dans l'usage, si ce n'est en France, du moins dans la francophonie. Car l'un des problèmes se situe là : la France étant souvent plus conservatrice ${ }^{11}$ voire plus hostile aux innovations que d'autres pays francophones, tout se passe comme si des formes peu légitimées dans l'Hexagone - pays de référence pour les professeur.e.s et élèves apprenant le français - du fait même qu'elles y sont regardées de façon suspecte, avaient du mal à se faire une place dans les ouvrages scolaires pour allophones, comme si on attendait que les natives et natifs 
fassent le premier pas pour que, à l'étranger, on se sente autorisé à décliner les professions au féminin en français.

L'Académie française qui, aux dires de la célèbre lexicographe feu Josette Rey-Debove, est pourtant aujourd'hui « une fausse référence puisqu'elle n'émet pas de pensée sur la langue $»^{12}$, a probablement encore une influence morale sur les auteur.e.s de méthodes de langue et ses propos, qui paraissent désormais dépassés, continuent cependant à faire autorité. Ainsi en va-t-il de sa «Mise au point» de $2014^{13}$, qui a fait suite à un incident s'étant produit à l'Assemblée nationale. Elle avait alors déclaré que " conformément à sa mission, défendant l'esprit de la langue et les règles qui président à l'enrichissement du vocabulaire, elle [rejetait] un esprit de système qui tend à imposer, parfois contre le vœu des intéressées, des formes telles que professeure, recteure, sapeuse-pompière, auteure, ingénieure, procureure, etc., pour ne rien dire de chercheure, qui sont contraires aux règles ordinaires de dérivation et constituent de véritables barbarismes ». Un tel discours ne pouvait que freiner les conceptrices et concepteurs de manuels. Une position qui, cinq ans plus tard, a changé, face à l'évolution inéluctable de la société et de l'usage. En février 2019, dans un rapport ${ }^{14}$ rédigé par une commission dirigée par Gabriel de Broglie, l'Académie s'est enfin résolue à accepter de nouveaux termes féminins relatifs aux titres et professions. Une avancée encore prudente, mais réelle. Il était grand temps. Il eut été difficile de continuer à stigmatiser des vocables désormais bien ancrés dans les habitudes des francophones.

Souhaitant aller dans le même sens et étant convaincue et convaincu de la nécessité de dépasser la timidité de certaines méthodes, nous avons opté pour une approche où la femme occupe la place qui lui revient dans la langue, partant du principe, à l'instar de Nicole Cholewka, que «ce qui n'est pas nommé n'existe pas (ou est considéré comme négligeable) »(Cholewka, 2000 : 313). Dans la lignée du principe proposé par Le franç@is dans le mouv' (Gaugey et Sheeren, 2015: 28-29), nous conseillons de proposer des exercices aux élèves où

- il est demandé d'appliquer les règles classiques de formation du féminin, mais en insérant des termes sémantiquement dissymétriques : le professionnel / la professionnelle, le chien / la chienne, le secrétaire / la secrétaire, un homme facile / une femme facile. Cet exercice, qui se veut préliminaire, permet de faire prendre conscience aux élèves du phénomène de péjoration. Mis au féminin, certains termes comportent malheureusement parfois une connotation négative, versant dans la sphère sexuelle.

- sont retranscrites des formulations sexistes à l'intérieur desquelles la femme est invisible ou presque (par exemple une phrase telle que «Le Conservateur du musée, Mme X, a invité les employés à venir au vernissage de l'exposition » et leur demander de rendre celle-ci plus présente à travers des modifications.

- l'inconnue à trouver n'est pas uniquement le féminin (afin de ne pas renforcer l'idée bien ancrée que le masculin est le genre normé, non marqué et régulier). Ceci implique également de changer la disposition habituelle d'un exercice en colonnes, par exemple, où l'on place le masculin à gauche et le féminin à droite, c'est-à-dire dans le sens où l'œil occidental est habitué à lire.

- sont proposés différents termes ayant été féminisés (suivant les règles de la morphologie ou non). L'exercice consiste à deviner celui qui est proposé par les guides de féminisation tels Mettre au féminin (2014, $3^{e}$ éd.) et Femme j'écris ton nom (1999). C'est en fait un choix multiple.

- il s'agit de demander aux élèves d'imaginer le «nouveau » féminin récemment entré dans la langue (sans leur fournir le néologisme officiel) et, une fois les propositions recueillies, de 
leur demander celui qu'ils/elles préfèrent, qui leur sonne le mieux. Il s'agit parfois simplement d'appliquer la règle de formation traditionnelle du féminin (contrôleur) contrôleuse, facteur/factrice), mais aussi de lancer un débat sur l'absence de vocable pour désigner celle qui exerce cette profession. visant à diffuser les nouveaux féminins, on contribue à faire prendre conscience du vide lexical qui caractérise parfois les professions exercées par les femmes et l'on propose une réflexion sur la langue. Peut-on agir sur celle-ci ou doit-elle évoluer naturellement ? Est-il normal qu'il n'existe pas de féminin pour les termes précurseur ou vainqueur? Quelle image vous vient à l'esprit lorsque vous entendez «le contrôleur arrive » ou « je vais chez le médecin $»^{15}$ ? Comment choisir entre sculpteure, sculpteuse ou sculptrice? Le fait de proposer un nouveau terme dans la langue pour qualifier une femme peut-il contribuer à lui conférer un statut? Est-ce que cela choque l'oreille? Savez-vous qu'il existe aussi de nouveaux masculins comme sage-homme ou maïeuticien?

\section{Décloisonner la division binaire du genre}

L'une des conséquences d'un enseignement traditionnel du genre est, d'une part, de proposer une vision extrêmement cloisonnée de celui-ci pour ce qui concerne les mots, d'autre part d'instaurer une frontière très rigide entre le masculin et le féminin de façon générale. Or, cette dichotomie est aujourd'hui remise en question, notamment par les mouvements LGBTQI ${ }^{16}$.

«La beauté de la féminisation réside dans sa variété et sa polyvalence. Différentes personnes non binaires, agenres, fluides dans le genre ou ayant un autre genre que strictement masculin ou féminin se servent de différentes stratégies de féminisation pour se désigner d'une façon qui les rende à l'aise, par exemple la rédaction épicène ou les graphies tronquées au singulier [...]. La féminisation, en reconnaissant que la langue doit être adaptée aux besoins des personnes et représenter la diversité des genres, peut servir à construire un discours respectueux des personnes non binaires, agenres, bigenres ou fluides dans le genre» (Lessard et Zaccour, 2018 : 22-23).

Voilà donc que les objectifs didactiques vont bien au-delà du simple fait d'enseigner à bien parler une langue. Ici, il s'agit de sensibiliser les apprenantes et apprenants au fait que l'identité sexuelle d'un individu peut être floue, non définie et que cette différence peut être reconnue et, par conséquent, légitimée dans le langage. Il n'est d'ailleurs plus rare de trouver certains formulaires administratifs où une troisième case à cocher est prévue outre la distinction habituelle M/F.

Afin de rompre la séparation entre le féminin et le masculin, nous proposons des activités où il est demandé aux étudiants et étudiantes de chercher le genre des mots en veillant à insérer :

- des mots épicènes (artiste, peintre, mannequin...).

- des termes ayant deux genres (après-midi, interview...), même s'il ne s'agit pas d'êtres animés.

- des mots dont la terminaison peut aider à deviner le genre afin de les sensibiliser aux suffixes et au genre auxquels ceux-ci sont généralement associés ou, à l'inverse, des mots 
dont la terminaison ne permet pas de donner une indication sur leur genre (oasis, équivoque).

- des vocables ressemblant à l'italien mais dont le genre est différent en français (armoire, rencontre, dent) et, pourquoi pas, des noms de pays. Ceci permet de contrer une forme d'ethnocentrisme qui caractérise souvent les esprits des jeunes qui croient que leur système linguistique est universel et à les sensibiliser à l'arbitraire du signe.

- des mots pouvant changer de genre quand ils sont au pluriel (amour, orgue, délice) ou pouvant changer de signification en fonction de l'article qui les précède (le/la page, un/une livre, le/la poste)

- des termes féminins se référant généralement à un homme (une sentinelle, une recrue, une vedette) ou des termes masculins se référant souvent à une femme (un mannequin, un souillon). Dans la même lignée, pourquoi ne pas fournir un tableau divisé en plusieurs colonnes à l'intérieur desquelles les apprenants/apprenantes devraient placer des termes dont le genre est masculin/féminin/épicène, mais aussi des termes féminins pouvant s'appliquer à un homme (une canaille, une brute, une star) ou le contraire (un témoin, le successeur)?

\section{Bouleverser certaines habitudes langagières}

La langue est constituée d'expressions toutes faites, de formules qui, à force d'être répétées, deviennent des sortes de clichés langagiers, des façons de parler fossilisées. Mis à part la formule Mesdames (Mesdemoiselles) Messieurs, force est de constater que dans les couples de mots (onomastique familiale, dénominations de groupes), le masculin précède toujours le féminin : le père et la mère / avoir des frères et sœurs / les hommes, femmes et enfants. Autant d'expressions fixées qui sont transmises de générations en générations. Nous proposons donc aux étudiantes et étudiants des phrases dans lesquelles nous inversons la disposition habituelle, de manière à les surprendre, à rompre les schèmes habituels de parole : ma tante et mon oncle / les lesbiennes et les gays, etc.

Nous préconisons également le procédé qui consiste à ne pas systématiquement enseigner les conjugaisons en négligeant les pronoms personnels sujets féminins. Bien qu'ils soient régulièrement signalés dans les méthodes, le je, tu, il, nous, vous, ils est parfois encore trop présent dans l'esprit des professeur/e/s. Le «on» est parfois négligé et les formes féminines sont souvent moins présentes que les formes masculines. Dès lors, il ne faut pas s'étonner que les étudiant.e.s (ou élèves) rencontrent des difficultés à utiliser "elle/elles" à l'oral, souvent remplacés par des formes masculines, à l'instar du pronom démonstratif neutre cela (abrégé en ça), très utilisé et sur lequel on insiste probablement trop peu si bien qu'il est souvent oublié.

41 On constate également que, dans des exercices comprenant un participe passé à accorder et où il manque un élément permettant de déterminer le genre de la personne à laquelle on se réfère, les étudiant.e.s ont tendance à systématiquement accorder au masculin. Ainsi en va-t-il d'un récit au passé composé à la première personne du singulier (je suis arrivé, je suis parti, le guide m'a conduit...) lorsque l'identité sexuelle de l'instance narrative n'est pas précisée. Il convient de signaler aux apprenant.e.s les deux solutions possibles à chaque fois. 


\section{La rédaction épicène, l'écriture inclusive} lourds que d'autres, demandent un peu plus d'efforts, manquent parfois de clarté ou de fluidité à l'écrit. D'autres au contraire passent inaperçus, semblent couler de source. Bref, il convient d'évaluer chacune de ces stratégies, de privilégier une certaine lisibilité et d'opter pour celle qui correspond le mieux aux intentions communicatives ou aux objectifs didactiques définis au préalable. Il convient également de varier ces techniques afin d'éviter une certaine monotonie mais aussi d'en montrer la pluralité. L'écriture inclusive est multiple et souple. La réduire au point médian, par exemple, serait une erreur. L'important est de rendre le genre féminin visible, quel que soit le procédé utilisé.

\section{Conclusion}

Notre article a voulu très succinctement présenter quelques procédés, assez simples à mettre en place, ayant pour objectif de proposer une autre vision de l'enseignement du genre, moins binaire, moins dichotomique que par le passé. Le véritable enjeu, selon nous, pour un renouvellement des interactions langagières, est bien de déritualiser nos habitudes normatives, les conscientiser et tenter de nouvelles approches, fondées socialement mais aussi didactiquement, résultats et analyses des performances de nos apprenant-e-s à l'appui. En somme, modifier nos comportements langagiers, ne pas reproduire celui que nous avons reçu en héritage et que nous tendons trop souvent à inculquer à notre tour aux enfants ou jeunes adultes. Si nous savons que le langage est 
le reflet d'une société, d'une vision du monde, nous savons également combien, à son tour, il peut influencer notre manière d'appréhender l'univers et de véhiculer des stéréotypes. Les enseignant-e-s jouent un rôle de premier plan dans ce processus de transmission. Il nous appartient de démonter ce cercle vicieux, le ce qui va de soi (cher à Barthes) et de le transformer en cercle vertueux en proposant une approche didactique qui évacue toute forme de sexisme et accorde aux femmes la place qui leur revient, en déconstruisant la tradition de manière à pouvoir donner place à l'innovation. C'est ce que nous avons tenté de faire dans cette contribution.

L'article dans son ensemble a été conçu à quatre mains. L'introduction et la conclusion, notamment, sont le fruit d'une double plume. La première partie du paragraphe 1 est due à Hugues Sheeren, la deuxième à Thérèse Manconi. Les paragraphes 2 (et ses sousparties) et 3 ont été rédigés par Thérèse Manconi tandis que les paragraphes 4,5, 6 et 7 ont été rédigés par Hugues Sheeren.

Académie française. (2014). La féminisation des noms de métiers, fonctions, grade ou titre Mise au point de l'Académie française. http://www.academie-francaise.fr/actualites/lafeminisation-des-noms-de-metiers-fonctions-grades-ou-titres-mise-au-point-delacademie Bargy, H. (1925). Description phonétique du présent du verbe. Paris : Les Belles lettres. Becquer, A.-M., Cerquiglini, B. (dir.), Cholewka, N., Coutier, M. \& Mathieu, M.-J. (1999). Femme, j'écris ton nom. Guide d'aide à la féminisation des noms de métiers, titres, grades et fonctions. Paris : La Documentation française.

Bidaud, F. (2012). Grammaire du français pour italophones. Seconda edizione. Utet Università, Novara : De Agostini Scuola SpA.

Cerquiglini, B. (2018). Le ministre est enceinte. Ou la grande querelle de la féminisation des noms. Paris : Seuil.

Cerquiglini, B., Corbeil, J.-C., Klinkenberg, J.-M. \& Peeters B. (2000). Le français dans tous ses états, Paris : Flammarion.

Charliac, L., \& Motron, A-C. (2015). Phonétique progressive du français, niveau

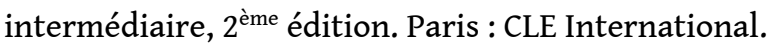

Cholewka, N. (2000). U comme us et pratiques de la langue, quelques aspects, ici et maintenant. Le français dans tous ses états. Paris : Flammarion.

Crimi, A. M., \& Hatuel, D. (2010). Vitamines : Méthode de français (1). Livre de l'élève et cahier. Recanati : ELI.

Delatour, Y., Jennepin D., Léon-Dufour M. \& Teyssier B. (2004). Nouvelle Grammaire du Français. Cours de Civilisation Française de la SORBONNE, Paris : Hachette.

Delbart, A-R. (2007). Jalons pour une grammaire instructionnelle de l'article français. Représentation du sens linguistique II. Bruxelles : De Boeck Supérieur.

Dister, A. \& Moreau, M.-L. (2020). Inclure sans exclure. Les bonnes pratiques de rédaction inclusive. Bruxelles : Direction de la Langue française - Service général des Lettres et du Livre - Fédération Wallonie-Bruxelles.

Dister, A. \& Moreau, M.-L. (2014). Mettre au féminin. Guide de féminisation des noms de métier, fonction, grade ou titre ( $3^{\mathrm{e}}$ édition). Bruxelles : Direction de la Langue française Service général des Lettres et du Livre - Fédération Wallonie-Bruxelles.

Doneux, J-L. (2001). L'écriture du français, Prédictibilité et Aléa. Publications de l'Université de Provence : Langues et Écritures.

Gaugey V., \& Sheeren H. (2015). Le franç@is dans le mouv'. Le lexique du français contemporain sous toutes ses coutures. Firenze : Le Lettere. 
Grégoire M. \& Thiévenaz O. (2017). Grammaire progressive du français (niveau intermédiaire A2/B1), Paris : CLE International.

Lenoble-Pinson, M. (2006). Chercheuse ? chercheur ? chercheure ? Mettre au féminin les noms de métier et les titres de fonction. Revue belge de philologie et d'histoire, 84 (3), 637-652.

Legros G., \& Moreau M.-L. (2012). Orthographe; qui a peur de la réforme ? Service de la langue française de la Fédération Wallonie-Bruxelles.

Magellan et Galilée - Questionner le monde CE2- éd. 2017, Hatier

Manconi, T. (2016). Une tentative de didactisation des avancées de la réflexion linguistique en FLM au profit du FLES. La description du verbe en français : entre morphologie et phonologie, in Digital Resources, creativity, innovative methodologies ans plurilinguism : new approaches to language teaching. Cambridge Scholars Publishing. Matthey, M. (2003). Apprentissage d'une langue et interaction verbale. Berne : Peter Lang. Moore, D., \& Simon, D-L. (2002). Déritualisation et identité d'apprenants. Acquisition et interaction en langue étrangère. http://aile.revues.org/1374.

Parodi L., \& M. Vallaco. (2003). Grammathèque, grammatica contrastiva per italiani, 2ème édition. Genova: Cideb Editrice.

Rabatel, A. \& Rosier, L. (2019). « Les défis de l'écriture inclusive », Le discours et la langue, 11 (1).

Riegel, M., Pellat, J-C., \& Rioul, R. (2004). Grammaire méthodique du français. Paris : PUF. Tétu, M. (1997). Qu'est-ce que la FRANCOPHONIE ?. Paris : Hachette-Edicef.

Van Raemdonck, D. et Detaille, M. (2010). Le sens grammatical - Référentiel à l'usage des enseignants. http://www.communelangue.com/

Viennot, E. (2014). Non, le masculin ne l'emporte pas sur le féminin ! Petite histoire des résistances de la langue française, Donnemarie-Dontilly : Éditions iXe.

Wilmet, M. (1998). Grammaire critique du français. Hachette-Duculot : EF AUT.

\section{NOTES}

1. Ce texte est conforme à la nouvelle orthographe.

2. On oppose, traditionnellement, grammaire descriptive (grammaire scientifique) et grammaire normative (grammaire scolaire ou ensemble de règles édictées selon le «bon usage »). Comme Marc Wilmet (1997), nous croyons que « l'exception infirme la règle » en lieu et place de l'adage normatif selon lequel « l'exception confirme la règle».

3. Il s'agit du manuel Magellan et Galilée - Questionner le monde, CE2 - éd. 2017, Hatier.

4. Contrairement à la politique habituelle de la revue qui réserve les guillemets uniquement aux citations, notre article en fera usage lorsque nous souhaitons marquer un effet de distanciation par rapport à certains termes.

5. https://www.change.org/p/nous-ne-voulons-plus-que-le-masculin-l-emporte-sur-lef\%C3\%A9minin

6. Les débats récents - et houleux - suscités suite à la publication d'une Tribune collective, le 18 octobre 2020, dans Marianne (https://www.marianne.net/agora/tribunes-libres/une-ecritureexcluante-qui-s-impose-par-la-propagande-32-linguistes-listent-les) et la réponse circonstanciée $\mathrm{du}$ linguiste Wagener (https://sysdiscours.hypotheses.org/155) ou encore d'un groupe de 65 
linguistes (https://blogs.mediapart.fr/les-invites-de-mediapart/blog/250920/au-dela-de-l-ecriture-inclusive-un-programme-de-travail-pour-la-linguistique-d-aujour) ne sauraient mieux illustrer la vivacité du thème ainsi que la nécessité - urgente, selon nous - d'aborder cela également sous l'angle de la didactique des langues (qu'elles soient premières, secondes ou étrangères).

7. Nous tenons à préciser que nous adopterons l'écriture inclusive dans le présent article, mais que les procédés auxquels

nous recourrons ne seront pas toujours identiques. Si nous avons conscience que le manque d'homogénéité peut déconcerter la lectrice ou le lecteur, nous estimons que figer l'écriture inclusive dans une seule stratégie formelle serait réducteur et en contradiction avec notre intention. Nous opterons donc pour différents procédés tout au long de cette contribution, afin de montrer combien l'écriture inclusive est souple et variée.

8. Ceci n'est qu'un exemple parmi tant d'autres, valable aussi pour (liste - hélas - non exhaustive) : Grammatica essenziale del francese (De Agostini, 2005); Grammaire facile du français (Zanichelli, 2005) ; Ça marche! Grammaire du français courant, (Loescher, 2008) ; Atelier de grammaire (Eli, Bordas et fils, 2011) ; Espace grammaire (Eli, Bordas et fils, 2011) ; et pour ne pas laisser entendre que le phénomène ne concerne « que » les maisons d'édition italiennes, un exemple qui vaut son "pesant d'or": Nouvelle grammaire du français - Cours de Civilisation française de la SORBONNE (Hachette, Français Langue étrangère, 2004).

9. Nous avions, dans un autre domaine et dans une autre contribution (Manconi, 2016), présenté une proposition de démarche didactique prenant en compte le morphologique/phonologique pour l'acquisition (à l'oral et à l'écrit) de l'indicatif présent du verbe en français, de la part d'apprenant.e.s allophones. Les travaux d'Henry Bargy (Bargy, 1925), en particulier son approche jeune de 95 ans de « description phonétique du présent du verbe » en constituaient le fondement. 10. Nous mettons en évidence, dans ce tableau, entre parenthèses obliques le(s) son(s) porteur(s) $\mathrm{du}$ « genre » masculin ou féminin, par contraste. Nous envisageons, à part, dans notre démarche les cas tels que « danseuse/danseur », « inspectrice/inspecteur », etc...

11. Déjà en 1997, Michel Tétu (Qu'est-ce que la FRANCOPHONIE ?) affirme ceci : « Après le Québec et la Suisse, la Belgique a décidé de féminiser plusieurs titres sans tenir compte de l'avis de la France trop conservatrice à son goût. La France continuera à dire Madame le directeur de la Bibliothèque alors que Belges, Suisses et Québécois diront Madame la directrice ». Lire également l'article «Le français contemporain » de Jacques Leclerc, collaborateur à la CEFAN, sur le site de l'Université Laval, où il déclare, à propos de la féminisation "Encore une fois, en matière de langue, la France semble se montrer frileuse, tant la tradition pèse lourdement ». https:// www.axl.cefan.ulaval.ca/francophonie/HIST_FR_s9_Fr-contemporain.htm

12. Voir interview dans la vidéo "Incorrigible orthographe" qui accompagne l'ouvrage Le français dans tous ses états (voir bibliographie) https://www.dailymotion.com/video/xmdn13. Une opinion corroborée par la brochure Orthographe : qui a peur de la réforme? conçue par le Service de la langue française de la Fédération Wallonie-Bruxelles où il est spécifié que "parce que le mandat de l'Académie ne correspond plus aux besoins actuels des sociétés, parce que le recrutement de ses membres ne tient pas compte de l'expertise qui s'est constituée et développée dans les sciences du langage, d'autres institutions chargées d'éclairer la politique linguistique ont été mises en place dans les différentes communautés francophones du Nord » (Legros-Moreau : 53).

13. http://www.academie-francaise.fr/actualites/la-feminisation-des-noms-de-metiersfonctions-grades-ou-titres-mise-au-point-de-lacademie

14. Lire à ce sujet https://www.lefigaro.fr/langue-francaise/actu-des-mots/ 2019/02/20/37002-20190220ARTFIG00028-dominique-bona-la-feminisation-permettra-aux-

femmes-de-sortir-d-un-malaise-linguistique.php

Recherches en didactique des langues et des cultures, 17-3 | 2020 
15. Cf. vidéo http://www.agirpourlegalite.org/lecriture-inclusive-ou-redaction-nondiscriminante-contre-le-sexisme-et-le-classisme/ utilisable en cours de langue pour travailler sur les représentations, et voir comment la langue façonne nos imaginaires.

16. Lesbiennes, gays, bis, trans, queers et intersexes. Longtemps appelé LGBT, le mouvement a ajouté deux lettres à son sigle depuis quelques années.

17. Nous n'enseignons pas de graphies tout à fait récentes comme les pronoms iels ou toustes, néologismes qui sont encore trop peu connus pour être enseignés en FLE.

18. http://www.lepoint.fr/societe/des-enseignants-ne-veulent-plus-appliquer-la-regle-lemasculin-l-emporte-sur-le-feminin-08-11-2017-2170713_23.php

\section{RÉSUMÉS}

Cette contribution vise à offrir des stratégies pédagogiques et didactiques pour rendre l'enseignement de la langue française non discriminatoire envers les femmes, y compris à travers quelques réflexions sur l'écriture inclusive. En partant d'exemples concrets faciles à mettre en place, l'article tente de démontrer qu'il est aisé de proposer une autre approche théorique (enseigner « le genre » et non plus « le féminin ») ainsi que certaines activités ayant pour objectif de décloisonner la dichotomie féminin vs masculin, l'idée étant que si l'on continue à enseigner une langue qui laisse les femmes de côté en les condamnant à l'invisibilité, on contribue à perpétrer une représentation traditionnelle et sexiste de celles-ci.

Questo nostro contributo intende proporre strategie pedagogiche e didattiche al fine di ascrivere l'insegnamento della lingua francese nelle riflessioni sociolinguistiche secondo le quali l'uso della lingua (e le scelte in quanto a forme) rappresenta uno strumento per evacuare le discriminazioni anche nella realtà. Partendo da esempi concreti, semplici da attuare, l'articolo tenta di dimostrare quanto semplice sia proporre un altro approccio teorico, teso a un maggiore equilibrio (insegnare « il genere » e non più « il femminile ») così come alcune attività il cui scopo è quello di scardinare la dicotomia femminile vs maschile. Con la convinzione che se si continua a insegnare la lingua occultando la posizione delle donne, negando loro una visibilità, si tramanda una rappresentazione tradizionalista e sessista delle stesse.

\section{INDEX}

Mots-clés : féminisation - sexisme linguistique - didactique du FLE - écriture inclusive

Keywords : feminization - linguistic sexism - didactic of the FLE (French as a foreign and secondary language) - inclusive writing

\section{AUTEURS}

\section{MANCONI THÉRÈSE}

Dipartimento di Lingue, Letterature e Culture Moderne (Université de

Bologne)

teresa.manconi[at]unibo.it 
Thérèse Manconi est professeure de français langue étrangère depuis 1992. Elle a enseigné dans plusieurs universités italiennes (Pavia, Insubria et Milano Statale) et, depuis 2019, au LILEC de l'Université de Bologne.

Elle a obtenu sa licence en philologie romane et son master en didactique du FLES à l'Université de Bruxelles. Ses principaux centres d'intérêt et de recherche touchent les pratiques didactiques, les TICE, le renouvellement des modèles de description/ prescription dans la didactique de la langue française, ainsi que les représentations en jeu dans les interactions en classe de langue. Elle a participé à de nombreux projets européens pour la valorisation du plurilinguisme et des identités " plurielles » à travers les autobiographies langagières.

\section{SHEEREN HUGUES}

Centre Linguistique de l'Université de Florence

hugues.sheeren[at]unifi.it

Hugues Sheeren est professeur de français langue étrangère depuis 1997. Il a travaillé dans plusieurs universités du nord de l'Italie (Bologne, Ferrare, Vérone) en tant que chargé de cours ou comme lecteur de langue française. Il est actuellement « collaborateur et expert linguistique » au Centre Linguistique de l'Université de Florence. Ses domaines d'intérêt et de recherche concernent principalement la didactique des langues, la variation linguistique dans l'enseignement du FLE et l'intercompréhension entre langues romanes (il est d'ailleurs membre du comité d'administration de l'APICAD). 